\title{
Applications of Commutator-Type Operators to $p$-Groups*
}

\author{
Gábor Lukács ${ }^{\dagger}$
}

November 9, 2018

\begin{abstract}
For a $p$-group $G$ admitting an automorphism $\varphi$ of order $p^{n}$ with exactly $p^{m}$ fixed points such that $\varphi^{p^{n-1}}$ has exactly $p^{k}$ fixed points, we prove that $G$ has a fully-invariant subgroup of $m$-bounded nilpotency class with $(p, n, m, k)$-bounded index in $G$. We also establish its analogue for Lie $p$-rings. The proofs make use of the theory of commutator-type operators.
\end{abstract}

\section{Introduction}

In this paper all groups and rings are finite, and $p$ is always a prime number.

\subsection{Brief Historical Background}

In [6] \& [4] E. I. Khukhro formulated the following two conjectures on the structure of $p$-groups admitting an automorphism of $p$-power order:

Conjecture 1.1. A finite p-group admitting an automorphism of order $p$ with $p^{m}$ fixed points contains a subgroup of $m$-bounded class with $(p, m)$ bounded index.

*2000 Mathematics Subject Classification 20D, 20F, 17B

${ }^{\dagger}$ I wish to thank the anonymous donors whose generosity towards the Technion enabled me to pursue my Master's studies and to do this research. I am also grateful to York University and AVI Fellowships for financial support. 
Conjecture 1.2. A finite p-group admitting an automorphism of order $p^{n}$ with $p^{m}$ fixed points contains a subgroup of $m$-bounded derived length with $(p, n, m)$-bounded index.

(We use the term " $(a, b, \ldots)$-bounded" for "bounded above by some function of $a, b, \ldots$ ".) R. Shepherd [12], C. R. Leedham-Green and S. McKay's [7] result gave a positive answer to Conjecture 1.1 when $m=1$. I. Kiming [5] gave a positive answer to Conjecture 1.2 in the case $m=1$. He proved that in this case there also exists a subgroup of class at most 2 with $(p, n)$-bounded index.

In [10] Yu. Medvedev made two conjectures, on Lie rings:

Conjecture 1.3. A Lie p-ring admitting an automorphism of order $p$ with $p^{m}$ fixed points contains a nilpotent subring of m-bounded class whose index is $(p, m)$-bounded.

Conjecture 1.4. A Lie p-ring admitting an automorphism of order $p^{n}$ with $p^{m}$ fixed points contains a solvable subring of m-bounded derived length whose index is ( $p, n, m)$-bounded.

Yu. Medvedev [10] proved that Conjecture 1.3 implies Conjecture 1.1 and Conjecture 1.4 implies Conjecture 1.2. In 11 Yu. Medvedev deduced Conjecture 1.3, hence gave a positive answer to Conjecture 1.1. In [2] A. JaikinZapirain proved Conjecture 1.4, and therefore gave a positive answer to Conjecture 1.2. For a wider historical background I refer to the Introduction of [10] (and [9]).

\subsection{On This Paper}

As we mentioned in [9], the main results of this paper are the following two theorems inspired by Conjecture 1.4 and Conjecture 1.2:

Theorem A. Suppose that $L$ is a Lie p-ring admitting an automorphism $\varphi$ of order $p^{n}$ with exactly $p^{m}$ fixed points, such that $\varphi^{p^{n-1}}$ has exactly $p^{k}$ fixed points. Then $L$ has a nilpotent fully-invariant ideal of $m$-bounded class which has $(p, n, m, k)$-bounded index in $L$. 
Theorem B. If a finite $p$-group $P$ admits an automorphism of order $p^{n}$ with exactly $p^{m}$ fixed points, such that $\varphi^{p^{n-1}}$ has exactly $p^{k}$ fixed points, then $P$ has a fully-invariant subgroup of $(p, n, m, k)$-bounded index which is nilpotent of $m$-bounded class.

These theorems give in some sense a stronger result ( $m$-bounded nilpotency class) than Conjectures 1.2 and 1.4, however we have to "pay" for that by involving an additional parameter $k$ into the bound for the index. We

always have $m \leq k$. Note that if $m=k$, Theorems $\mathrm{A}$ and $\mathrm{B}$ are obvious consequences of Medvedev's theorems mentioned above. But in the case where $m<k$, we obtain a much better bound for the class ( $m$-bounded).

In Section 3 we prove Theorem A. The proof makes use of the framework of commutator-type operators, which we developed in [9], especially Theorem III (to which we will refer in this paper as "Theorem III"). It follows the design of Khukhro's version [3, 14.2] of a proof of a theorem of Medvedev (Conjecture 1.3) mentioned above.

In Section 1 we prove that Theorem B follows from Theorem A. The proof is done in a similar way as suggested by the referee in [10].

The experienced reader may wish to omit Section 2 (except for notation and definitions) and jump directly to Section 3.

\section{Preliminary Facts}

For the convenience of the reader and further reference we mention some results and notations which will be used occasionally in the paper.

\subsection{Automorphisms of Abelian Groups}

For any positive integer $k$ we denote by $\phi_{k}(x)$ the cyclotomic polynomial of order $p^{k}$.

Let $G$ be a group and $\varphi$ an automorphism of the group. The set of fixed points of $\varphi$ on $G$ is $C_{G}(\varphi)$.

Let $A$ be a finite additive $p$-group, that admits an automorphism $\varphi$ of order $p^{n}$ with exactly $p^{m}$ fixed points. 
Proposition 2.1. [8, 3.2] We have

$$
a+a \varphi+a \varphi^{2}+\cdots+a \varphi^{p^{n}-1}=a \prod_{k=1}^{n} \phi_{k}(x)=0
$$

for all $a \in p^{m} A$.

Lemma 2.2. [9, 3.2] Suppose that $\varphi$ satisfies (1) on A. Then:

(a) for any $\varphi$-invariant section $U$ of $A$ we have $p^{n} C_{U}(\varphi)=0$;

(b) for any homocyclic $\varphi$-invariant section $V$ of $A$ of exponent $p^{s}$ we have $\left|C_{V}(\varphi)\right|=\left|C_{p^{i} V / p^{i+n} V}(\varphi)\right|$ whenever $0 \leq i \leq s-n$.

Lemma 2.3. [9, 3.4] Let $A$ be an abelian group. If $\phi_{n^{\prime}}(\varphi)=0$ and $\varphi$ has order $p^{n}$ on $A$ then either $n^{\prime}=n$ or $n^{\prime}>n$. If $n^{\prime}>n$, then $p A=0$.

\section{$2.2 \quad$ Lie Rings}

Proposition 2.4. [3, 7.20] Let $L$ be a Lie ring of derived length 2, admitting an automorphism $\varphi$ of order $p$. Then $\gamma_{p+1}(p L) \subseteq$ id $\left\langle C_{L}(\varphi)\right\rangle$.

A Lie ring is called Lie $p$-ring if its additive structure is a $p$-group.

\section{Proof of Theorem $A$}

The proof of Theorem A follows the design of [3, 14.2]: we split it up into two theorems (Theorem 3.1 and Theorem 3.2 below). Then, in subsection 3.3 we show that they imply Theorem A. It is interesting to note that the tool of commutator-type operators we developed in [9] gives a common framework for proving Theorem 3.1 and Theorem 3.2.

\section{$3.1 m$-Bounded Derived Length}

Theorem 3.1. Suppose that L is a Lie p-ring. If L admits an automorphism $\varphi$ of order $p^{n}$ with exactly $p^{m}$ fixed points, such that $\varphi^{p^{n-1}}$ has exactly $p^{k}$ fixed points, then $L$ has a soluble fully-invariant ideal of $m$-bounded derived length which has $(p, n, m, k)$-bounded index in $L$. 
Proof. $\varphi^{p^{n-1}}$ has $p^{k}$ fixed points, thus by Proposition $2.1 \varphi^{p^{n-1}}$ satisfies the polynomial $\phi_{1}(x)=0$ on $p^{k} L$ (for its order is $p$ ). This means that $\phi_{1}\left(\varphi^{p^{n-1}}\right)=\phi_{n}(\varphi)=0$ on $p^{k} L . p^{k} L$ has $(p, n, m, k)$-bounded index in $L$ (because $L$ has $(p, n, m)$-bounded rank, see [3, 2.7]), hence replacing $L$ by $p^{k} L$ we may assume that $\phi_{n}(\varphi)=0$ on $L$. We note that this is the only step where $k$ plays any role. Later we will have $(p, n, m)$-bounded index.

If $R$ is a Lie-ring, clearly $T\left(A_{1}, A_{2}\right)=\left[A_{1}, A_{2}\right]$ is a commutator-type operator in 2 variables on $R$.

Let $\mathcal{L}$ be the set of all the triplets consisting of a finite Lie $p$-ring and the Lie bracket $[\cdot, \cdot]$ as a commutator-type operation in 2 variables and an automorphism $\psi$ of $L$ of $p$-power order.

We show that $\mathcal{L}$ satisfies condition (c) of Theorem III (clearly $\mathcal{L}$ is closed under "taking" $(T, \psi)$-invariant sections). Let $(R, T, \varphi) \in \mathcal{L}$ such that $R$ is homocyclic and admits an automorphism $\varphi$ of order $p^{n}$ with $p^{m}$ fixed points, such that $\phi_{n}(\varphi)=0$. Define $[[\cdot, \cdot]]$ as in [8, 2.14] (or [3, 13.24]) (with $t$ in place of $s$ ). Let $\widetilde{R}$ be the "lifted" ring, and let $\mathbb{T}$ be the "lifted operator" that $T$ defines on $\widetilde{R}$ (see 9 , page 15$]$ ). For $A, B \leq R$, clearly $\mathbb{T}(\widetilde{A}, \widetilde{B})=[[\widetilde{A}, \widetilde{B}]]$, because by the definion of $[[\cdot, \cdot]]$ we have $p^{t}[[A, B]]=[A, B]=T(A, B)$. Hence $\gamma_{u}(\widetilde{R})=\gamma_{u}^{\mathbb{T}}(\widetilde{R})$. By Proposition [3, 13.26], $\widetilde{R}$ forms a Lie-ring with this new multiplication. (All these are true, because the "classical" top of $R$ used in [3] coincides with $t(R)$ that $T$ defines.)

$\phi_{n}(\varphi)=0$ on $R$, thus $\phi_{n}(\varphi)=0$ on $\widetilde{R}$ (since it is a $\varphi$-invariant section of $R$ ). $\quad p C_{\widetilde{R}}\left(\varphi^{p^{n-1}}\right)=0$, by Lemma 2.2 applied to $\varphi^{p^{n-1}}$ (because $\varphi^{p^{n-1}}$ satisfies $\phi_{1}(x)=0$ on $\widetilde{R}$ ). Let $h=h(p)$ (the Higman number). By Higman's theorem, we have

$$
p^{h+2} \gamma_{h+1}(\widetilde{R})=p \gamma_{h+1}(p \widetilde{R}) \subseteq p_{\text {id }}\left\langle C_{\widetilde{R}}\left(\varphi^{p^{n-1}}\right)\right\rangle={ }_{\text {id }}\left\langle p C_{\widetilde{R}}\left(\varphi^{p^{n-1}}\right)\right\rangle=0 .
$$

Thus, for $u(p, n, m)=h(p), v(p, n, m)=h(p)+2, \mathcal{L}$ satisfies the assumptions of Theorem III. Note, that in this step we made use of the fact that $\widetilde{R}$ is also a Lie-ring (otherwise we could not apply Higman's theorem).

Therefore, by Theorem III, there exist an $m$-bounded number $g=g(m)$ and a $(p, n, m)$-bounded number $r=r(p, n, m)$, such that for any Lie $p$-ring $L$ admitting an automorphism $\varphi$ of order $p^{n}$ with $p^{m}$ fixed points such that $\phi_{n}(\varphi)=0$, we have $\left(p^{r} L\right)^{(g)}=T^{(g)}\left(p^{r} L\right)=0 . \quad p^{r} L$ has $(p, n, m)$-bounded index in $L$ (because the rank of $L$ and $r$ are $(p, n, m)$-bounded).

This completes the proof of Theorem 3.1. 


\section{$3.2 m$-Bounded Nilpotency Class}

To shorten the notation, we will write $\left[C,{ }_{i} D\right]=[C, \underbrace{D, \ldots, D}_{i \text { times }}]$.

Theorem 3.2. Let $L=A \oplus B$ be a Lie p-ring, with abelian ideal $A$ and abelian subgroup $B$. Suppose that $L$ admits an automorphism $\varphi$ of order $p^{n}$ with exactly $p^{m}$ fixed points, such that $A$ and $B$ are $\varphi$-invariant and $\varphi^{p^{n-1}}$ has exactly $p^{k}$ fixed points on L. Then there exits a $(p, n, m, k)$-bounded number $f=f(p, n, m, k)$ and an $m$-bounded number $g=g(m)$ such that $p^{f}\left[A,{ }_{g} B\right]=0$.

P Roof. Since the order of $\varphi^{p^{n-1}}$ is $p$, by Proposition 2.1 applied on $\varphi^{p^{n-1}}$ we obtain that $\phi_{1}\left(\varphi^{p^{n-1}}\right)=\phi_{n}(\varphi)=0$ on $p^{k} L$. Since

$$
\left[p^{k} A, g(m)\left(p^{k} B\right)\right]=p^{(g(m)+1) k}[A, g(m) B],
$$

replacing $L$ by $p^{k} L$ we may assume that $\phi_{n}(\varphi)=0$ on $L$ from the outset. We note that this is the only step where $k$ plays any role. Later we will have $(p, n, m)$-bounded index.

Let $\mathcal{A}$ be the set of the triplets $(A, T, \psi)$ such that $T$ is the operator $T(C)=[C, B]$ on $A$, where $[\cdot, \cdot]$ is the Lie-bracket in a Lie $p$-ring $L=A \oplus B$ (so $A$ and $B$ are $p$-groups), $A$ is an abelian $\psi$-invariant ideal in $L, B$ is an abelian $\psi$-invariant subring of $L$ and $\psi$ is an automorphism of $L$ such that $\phi_{n^{\prime}}(\psi)=0$ on $L$ for some $n^{\prime}$. From $\phi_{n^{\prime}}(\psi)=0$ it follows that $\psi^{p^{n^{\prime}}}=1$, so $\psi$ has $p$-power order.

We claim that $\mathcal{A}$ satisfies the conditions of Theorem III. First, suppose that $(A, T, \psi) \in \mathcal{A}$ and $S=C / D$ is a $(T, \psi)$-invariant section of $A$. Then $S$ is " $B$-invariant" (i.e. $[S, B] \leq S$ ), thus $C$ and $D$ are $B$-invariant, hence $D$ is an ideal of $C \oplus B$. So $S \oplus B$ forms a new Lie-ring, being a Lie-section of $L$ (a subring modulo its ideal). Clearly $(S, T, \psi) \in \mathcal{A}$.

Let $(A, T, \varphi) \in \mathcal{A}$ such that $A$ is homocyclic, $\left|C_{A}(\varphi)\right|=p^{m}$ and the order of $\varphi$ on $L$ is $p^{n}$. Let $\widetilde{L}=\widetilde{A} \oplus B$ with the operation $[[\cdot, \cdot]]$ : for any $x \in A$ and $y \in B$ we put $[[x, y]]$ to be a $p^{t}$ th root of $[x, y]$, where $t=t(A)$. If either $z_{1}, z_{2} \in A$ or $z_{1}, z_{2} \in B$ we define $\left[\left[z_{1}, z_{2}\right]\right]=0$. The additive factor group $\widetilde{L}$ endowed with this operation $([[\widetilde{x}, \widetilde{y}]]=[[\widetilde{x, y}]]$, where tilde denotes image in $\widetilde{L})$, is a Lie ring, and the automorphism of the additive group of $L$ induced by $\varphi$ is an automorphism of the Lie ring $\widetilde{L}$ (see Proposition [8, 6.7, page 41]). 
We show now that condition (c) of Theorem III holds here. Since $\widetilde{A}$ and $B$ are abelian and $\widetilde{A}$ is an ideal in $\widetilde{L}$, we have $\gamma_{u+1}(\widetilde{L})=\left[\left[A,{ }_{u} B\right]\right]$. Clearly $\mathbb{T}(C)=[[C, B]]$ for any $C \leq \widetilde{A}$, thus

$$
\gamma_{u+1}(\widetilde{L})=\gamma_{u+1}^{\mathbb{T}}(\widetilde{A}) .
$$

By the definition of $\mathcal{A}, \phi_{n^{\prime}}(\varphi)=0$ on $L$. By Lemma 2.3, if $n \neq n^{\prime}$, then $p L=0$, so we are done. Thus we may assume that $n=n^{\prime}$. As an abelian group, $\widetilde{L}$ is a $\varphi$-invariant section of $L$, hence $\phi_{n}(\varphi)=0$ on $\widetilde{L}$. Let $\sigma=\varphi^{p^{n-1}}$ (as an automorphism of $\widetilde{L}$ ). Then $\phi_{1}(\sigma)=0$, thus (applying Lemma 2.2(a) on $\sigma$ )

$$
p C_{\widetilde{L}}(\sigma)=0
$$

The "lifted" Lie-ring $\widetilde{L}$ has derived length 2, hence by Proposition 2.4 we have $\gamma_{p+1}(p \widetilde{L}) \subseteq$ id $\left\langle C_{\widetilde{L}}(\sigma)\right\rangle$. Hence,

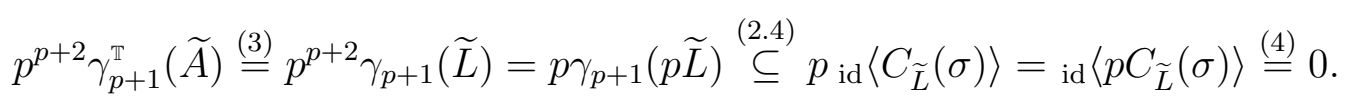

Therefore, $\mathcal{A}$ satisfies the conditions of Theorem III with $v(p, n, m)=p+2$, $u(p, n, m)=p$.

So there exist a $(p, n, m)$-bounded number $f(p, n, m)$ and an $m$-bounded number $g(m)$ such that for any $L=A \oplus B$ such that $\phi_{n}(\varphi)=0$ on $L$ and $\varphi$ has at most $p^{m}$ fixed points on $\boldsymbol{A}$, then $T^{(g)}\left(p^{f} A\right)=p^{f} T^{(g)}(A)$ (in particular, it is true if $\left.\left|C_{L}(\varphi)\right|=p^{m}\right)$.

This completes the proof of Theorem 3.2.

\subsection{Theorem [3.1] and 3.2 imply Theorem $A$}

In this subsection we will show, how Theorem A follows from Theorem 3.1 and Theorem 3.2. First note the following corollary of Theorem 3.2 .

Corollary 3.3. Let $L$ be a soluble Lie p-ring of derived length 2. Suppose that $L$ admits an automorphism $\varphi$ of order $p^{t}$ with exactly $p^{s}$ fixed points, such that $\varphi^{p^{t-1}}$ has $p^{r}$ fixed points. Then $\gamma_{g(s)}\left(p^{f(p, t, s, r)} L\right)=0$ for $a(p, t, s, r)$ bounded number $f(p, t, s, r)$ and an s-bounded number $g(s)$.

This corollary is an adaption of [3, 14.39] to our conditions. 
Proof. Put $A=[L, L]$ and $B=L /[L, L]$. We define the (Lie) operation of $A \oplus B$ as follows: if both $x, y$ in $A$ or in $B$, then $[x, y]=0$, and if $y \in B$ then $[a, b]=[a, y]$ if $b=y+[L, L] \in B . A$ and $B$ are $\varphi$-invariant, and we have $\left|C_{A \oplus B}(\varphi)\right|=p^{m} \leq p^{2 s}$, and $\left|C_{A \oplus B}\left(\varphi^{p^{t-1}}\right)\right|=p^{k} \leq p^{2 r}$ (see [3, 2.12]). It follows form the definition of $L$, that $A$ is an abelian ideal in $A \oplus B$ and $B$ is an abelian subring, and they are $\varphi$-invariant.

If $\varphi$ has order less than $p^{t}$ on $A \oplus B$, then $\varphi^{p^{t-1}}=1$ on $A \oplus B$, so we have $p^{2 r}[L, L]=0$ (for $\left|C_{A \oplus B}\left(\varphi^{p^{t-1}}\right)\right| \leq p^{2 r}$ ), and we are done. So we may assume that $\varphi$ has order $p^{t}$ on $A \oplus B$. Thus $A \oplus B$ satisfies the conditions of Theorem 3.2, with parameters $t, m \leq 2 s$ and $k \leq 2 r$. Hence for some $s$-bounded number $v$ and $(p, t, s, r)$-bounded number $u$ we have $p^{u(p, t, s, r)}\left[A,_{v(s)} B\right]=0$. ( $m$ and $k$ appear in the bounds given by Theorem 3.2, but they are $s$ and $r$ bounded respectively and the bounds can be assumed to be monotonic functions). By the definition of the operation in $A \oplus B$, it implies that

$$
\gamma_{v+1}\left(p^{[u /(v+1)]+1} L\right) \leq p^{u}\left[[L, L],{ }_{v(s)} L\right]=p^{u(p, t, s, t)}\left[A,_{v(s)} B\right]=0 .
$$

This completes the proof of Corollary 3.3 .

Proposition 3.4. Suppose that $L$ is a soluble Lie p-ring of derived length $d$. If $L$ admits an automorphism $\varphi$ of order $p^{n}$ with exactly $p^{m}$ fixed points, such that $\varphi^{p^{n-1}}$ has $p^{k}$ fixed points on $L$, then $L$ has a fully-invariant nilpotent ideal of $(m, d)$-bounded class which has $(p, n, m, k, d)$-bounded index in $L$.

This proposition is an adaption of [3, 14.40] to our conditions.

Proof. We proceed by induction on $d$, the derived length of $L$. If $d=2$, the result follows from Corollary 3.3. Suppose that $d>2$. By the inductive hypothesis, $\gamma_{v}\left(p^{u}[L, L]\right)=0$ for $u=u(p, n, m, k, d-1)$ and $v=v(m, d-1)$. We have $\left|C_{[L, L]}\left(\varphi^{p^{n-1}}\right)\right|,\left|C_{L / L^{(2)}}\left(\varphi^{p^{n-1}}\right)\right| \leq\left|C_{L}\left(\varphi^{p^{n-1}}\right)\right|$ (see [3, 2.12]), so by Corollary 3.3 (applied to $\left.L / L^{(2)}\right), \gamma_{g}\left(p^{f} L\right) \leq L^{(2)}$ for some $g=g(m)$ and $f=f(p, n, m, k)$. We put $w=\max \{[u / 2]+1, f\}$, which is $(p, n, m, k, d)$ bounded, and put $M=p^{w} L$. Then

$$
\begin{aligned}
\gamma_{g+4}(M) & =\gamma_{g+4}\left(p^{w} L\right) \leq\left[L^{(2)}, p^{w} L, p^{w} L, p^{w} L, p^{w} L\right] \\
& \leq p^{4 w} L^{(2)}=\left(p^{w} L\right)^{(2)}=M^{(2)}
\end{aligned}
$$

therefore $\gamma_{g+4}(M) \leq M^{(2)}$. 
But since $u \leq 2 w, \gamma_{v}([M, M])=\gamma_{v}\left(p^{2 w}[L, L]\right) \leq \gamma_{v}\left(p^{u}[L, L]\right)=0$. Applying [0, 5.27] with $L=M$ and $N=[M, M]$ we obtain that the nilpotency class of $M$ is bounded in the terms of $v$ and $g$, that is $(m, d)$-bounded. Thus, $M$ is the required fully-invariant ideal of $(m, d)$-bounded class with $(p, n, m, k, d)$ bounded index in $L$.

Proof of Theorem A. According to Theorem 3.1, $L$ has a soluble fully-invariant ideal of $m$-bounded derived length with $(p, n, m, k)$-bounded index in $L$. Thus, without loss of generality we may assume that $L$ has $m$ bounded derived length. By Proposition 3.4, $L$ has a nilpotent fully-invariant ideal of $(m, d)$-bounded class with $(p, n, m, k, d)$-bounded index in $L$. Since $d$ is $m$-bounded, the result follows.

This completes the proof of Theorem 3.1.

\section{Proof of Theorem B}

The structure of the proof of Theorem B follows the scheme suggested by the referee in 10.

Lemma 4.1. If $G$ satisfies the conditions of the Theorem, then there exist $a(p, n, m, k)$-bounded number $f_{1}=f_{1}(p, n, m, k)$ and an $m$-bounded number $g=g(m)$ such that $\gamma_{g}\left(G^{f^{f_{1}}}\right) \subseteq \gamma_{p}(G)$.

Proof. First suppose that, $G$ is of class $<p$. Let $L$ be the corresponding Lie-ring, by the Lazard correspondence [3, chapter 10, 10.24] (inverse Baker-Hausdorff formula). $L$ fulfils the conditions of Theorem A, so we have $\gamma_{g(m)}\left(p^{f_{1}(p, n, m, k)} L\right)=0$ for the $(p, n, m, k)$-bounded number $f_{1}$ and the $m$-bounded number $g$. The correspondent of $p^{f_{1}(p, n, m, k)} L$ is $G^{p^{f_{1}(p, n, m, k)}}$, so $\gamma_{g}\left(G^{p^{f_{1}}}\right)=1$.

Now suppose that $G$ is an arbitrary group satisfying the conditions of the theorem. Then, applying the consideration above to $\bar{G}=G / \gamma_{p}(G)$ we obtain that $\gamma_{g}\left(G^{p^{f_{1}}}\right) \gamma_{p}(G) / \gamma_{p}(G)=\gamma_{g}\left(\bar{G}^{p^{f_{1}}}\right)=1$. Therefore $\gamma_{g}\left(G^{p^{f_{1}}}\right) \subseteq \gamma_{p}(G)$, as desired.

Proof of Theorem B. Let $\psi=\varphi^{p^{n-1}}$, then $\left|C_{P}(\psi)\right|=p^{k}$. P has a fully invariant subgroup of $(p, k)$-bounded index which is nilpotent of class at most $h(p)$ (see [3, 8.1]; it follows from the proof that the characteristic 
subgroup found in the theorem is, in fact, fully-invariant). Thus, we may assume that

$$
P \text { is nilpotent of class at most } h(p) \text {. }
$$

Let $g=g(m)$ and $f_{1}=f_{1}(p, n, m, k)$ from Lemma 4.1 applied to $P$. We show that there exists a $(p, n, m, k)$-bounded number $f=f(p, n, m, k)$, such that $\gamma_{g}\left(P^{p^{f}}\right)=1$. If $p \leq g(m)$, then the class of $P$ is $m$-bounded (by (5)). If $p>g(m)$, we proceed by induction on $c$, the class of $P$. By Lemma 4.1, $\gamma_{g}\left(P^{f_{1}}\right) \subseteq \gamma_{p}(P)$. So $\gamma_{c-p+g}\left(P^{f^{f_{1}}}\right) \subseteq \gamma_{c}(P)=1$. Since $g<p, c-p+g<c$, thus by the inductive hypothesis there exists a $(p, n, m, k)$-bounded number $f_{2}(p, n, m, k)$ such that $\gamma_{g}\left(\left(P^{p^{f_{1}}}\right)^{p^{f_{2}}}\right)=1$.

Let $f=f_{1}+f_{2}$. Since $P^{p^{f_{1}+f_{2}}} \subseteq\left(P^{p^{f_{1}}}\right)^{p^{f_{2}}}$, we have $\gamma_{g}\left(P^{p^{f}}\right)=1$. Clearly $P^{p^{f}}$ is fully-invariant. Let $H=P / P^{p^{f}}$. By Burnside Theorem, the number of generators of $H$ is the same as the number of generators of $K=H / \Phi(H)$ (here $\Phi(H)=H^{p}[H, H]$ is the Frattini subgroup of $H$ ). $K$ is an abelian $\varphi$-invariant section of $H$ with $\left|C_{K}(\varphi)\right| \leq p^{m}$ (see [3, 2.12]). Hence (by Corollary [3, 2.7]), $r(K) \leq m p^{n}$, so $H$ has at most $m p^{n}$ generators. The exponent of $H$ is at most $p^{f}$. Thus the exponent, the number of generators and the nilpotency class of $H$ are $(p, n, m, k)$-bounded. Therefore, the order of $H$ is $(p, n, m, k)$-bounded (see [3, 6.12(c)]), hence $P^{p^{f}}$ has $(p, n, m, k)$-bounded index in $P$. By (可) $c \leq h(p)$, thus we have $p$-bounded number of steps in the induction.

This completes the proof of Theorem B.

\section{Acknowledgements}

I am deeply indebted to my Master's thesis supervisor, Prof. Arye Juhász, for his dedicated mentorship that made this research possible.

I am grateful to Prof. Pavel Shumyatsky, who gave me preprints of the papers [10] and [11] of Medvedev in February 1999, before their publication.

I would like to thank to Prof. Walter Tholen, who was of great assistance in editing the paper - both in its formation, articulation, and preparation for submission. 
I would like to express my heartfelt gratitude to my father, Dr. János Lukács, for providing me with a secure home environment making possible the flowering of this research.

Last, but not least, special thanks to my students for their enormous encouragement.

\section{References}

[1] J. L. Alperin, Automorphisms of solvable groups, Proc. Amer. Math. Soc. 13 (1962), 175-180.

[2] A. Jaikin-Zapirain, On almost regular automorphisms of finite p-groups, Advances in Mathematics 153, 391-402 (2000).

[3] E. I. Khukhro, p-Automorphisms of Finite p-Group, volume 246 of London Mathematical Society: Lecture note series. Cambridge University Press, Cambridge, 1998.

[4] E. I. Khukhro, Finite p-groups admitting p-automorphisms with few fixed points, Mat. Sb. 184 (1993), 53-64 (Russian); English translation, Russian Acad. Sci. Sbornik Math, 80 (1995), 435-444.

[5] I. Kiming, Structure and derived length of finite p-groups possessing an automorphism of p-power order having exactly $p$ fixed points, Math. Scand. 62 (1988), 153-172.

[6] The Kourovka Notebook: unsolved problems in group theory, 10th ed., Inst. Math. Sibirsk. Otdel. Akad. Nauk SSSR, Novosibirsk, 1986.(Russian)

[7] C. R. Leedham-Green and S. McKay, On p-groups of maximal class I, Quart. J. Math. Oxford (2) 27 (1976), 297-311.

[8] G. Lukács, Master's Thesis, Technion - Israel Institute of Technology, 1999, http://www.math.yorku.ca/ lukacs/MAthesis

[9] G. Lukács, Commutator-Type Operators Preprint, York University, Toronto, 1999. 
[10] Yu. Medvedev, p-Groups, Lie p-Rings and p-Automorphisms, J. London Math. Soc. (2) 58 (1998), 27-37.

[11] Yu. Medvedev, p-Divided Lie Rings and p-Groups J. London Math. Soc. (2) 59 (1999), 787-798.

[12] R. Shepherd, Ph.D. Thesis, University of Chicago, 1971.

Department of Mathematics \& Statistics

York University, 4700 Keele Street

Toronto, Ontario, M3J 1P3

Canada

e-mail: lukacs@mathstat.yorku.ca 